\title{
Improved Hamiltonian Lattice Gauge Theory
}

\author{
J. Carlsson ${ }^{\mathrm{a} *}$, J. A. L. McIntosh ${ }^{\mathrm{a}}$, B. H. J. McKellar and L. C. L. Hollenberg ${ }^{\mathrm{a}}$

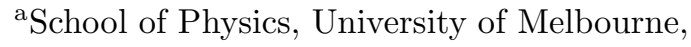 \\ Victoria 3010, Australia.
}

We derive an improved lattice Hamiltonian for pure gauge theory, coupling arbitrarily distant links in the kinetic term. The level of improvement achieved is examined in variational calculations of the SU(2) specific heat in $2+1$ dimensions.

\section{Introduction}

To date, the majority of work in lattice QCD has been performed in the action formulation. An advantage of this approach is that it readily lends itself to Monte Carlo techniques. Working in the Hamiltonian approach [1] brings a different intuition to the problem and serves as a check of universality. An advantage of Hamiltonian lattice gauge theory is in the applicability of techniques from many body physics [2]. Also, it appears that in finite density QCD, a Hamiltonian approach is favorable due to the so-called complex action problem which rules out the use of standard Monte Carlo techniques in the action formulation [3].

Much work in the past decade has been devoted to improving lattice actions 四. In contrast, the improvement of lattice Hamiltonians has only recently begun. Perhaps the most extensive treatment to date is due to Luo, Guo, Kröger and Schütte [5] who discussed the improvement of Hamiltonian lattice gauge theory for gluons. In their study it was discovered that deriving an improved Hamiltonian from a Symanzik improved action, whether by transfer matrix or canonical Legendre transformation, results in a kinetic Hamiltonian with an infinite number of terms coupling lattice sites which are arbitrarily far apart. To derive a local kinetic Hamiltonian coupling only nearest neighbor lattice sites it was found necessary to start with an improved action

\footnotetext{
*j.carlsson@physics. unimelb.edu.au

${ }^{\dagger}$ Presenter at the conference.
}

with an infinite number of terms, coupling distant lattice sites.

With this technique the order $a^{2}$ errors are removed from the Kogut-Susskind Hamiltonian. However, generating Hamiltonians with further improvement would seem exceedingly difficult. This is because one would need to start from a Lüscher-Weisz improved action with non-planar terms [6]. For this reason we propose a move to the Symanzik approach, as applied to the Hamiltonian. That is, in the spirit of the original Kogut-Susskind paper, to construct improved Hamiltonians directly by adding appropriate gauge invariant terms and fixing their coefficients so that errors are canceled.

\section{Symanzik Improvement of the Lattice Hamiltonian}

The Kogut-Susskind Hamiltonian for pure $\mathrm{SU}(N)$ gauge theory on the lattice is given by

$H_{0}=\frac{a^{3}}{2} \sum_{x, i} \operatorname{Tr}\left[E_{i}^{L}(x)^{2}\right]+\frac{2 N}{a g^{2}} \sum_{x, i<j} P_{i j}(x)$.

Here $E^{L}$ is the lattice chromo-electric field, $N$ is the dimension of the gauge group, and $P_{i j}(x)$ is the usual plaquette and operator in the $(i, j)$ plane.

To improve the potential part of the KogutSusskind Hamiltonian, we follow the process of improving the Wilson action. By introducing the usual rectangle operator $R_{i j}(x)$ in the $(i, j)$ plane (with the long side in the $i$ direction), and expanding in powers of $a$, we arrive at the order $a^{2}$ 
improved potential term:

$V_{2}=\frac{2 N}{a g^{2}} \sum_{x, i<j}\left\{\frac{5}{3} P_{i j}(x)-\frac{1}{12}\left[R_{i j}(x)+R_{j i}(x)\right]\right\}$.

To improve the kinetic part of the Kogut Susskind Hamiltonian we proceed as follows. The lattice gluon field $A_{\mu}^{L}$ is defined to be the average of the continuum gluon field $A$ along the link joining $x$ and $x+a \mu$ :

$A_{\mu}^{L}\left(x^{\prime}\right)=\frac{1}{a} \int_{\text {Link }} d x \cdot A \Rightarrow U_{\mu}(x)=e^{i g a A_{\mu}^{L}\left(x^{\prime}\right)}$,

where $x^{\prime}$ is a point near the points $x$ and $x+a \mu$. On the lattice, the gluon field is defined at only one point along (or nearby) a link. This leads to interpolation errors in in Eq. (3). For instance, by choosing to evaluate the gluon field at the midpoint of the link, the lattice and continuum fields are related by

$A_{\mu}^{L}(x) \approx A_{\mu}(x)+\frac{a^{2}}{24} \partial_{\mu}^{2} A_{\mu}(x)+\frac{a^{4}}{1920} \partial_{\mu}^{4} A_{\mu}(x)$.

Since the electric field must generate group transformations, $\left[E_{i}^{\alpha}(x), A_{j}^{\beta}(y)\right]=-i \delta_{x y} \delta_{i j} \delta_{\alpha \beta} / a^{3}$, a similar relation between the lattice and continuum electric fields can be derived:

$E_{i}^{L \alpha}(x) \approx E_{i}^{\alpha}(x)-\frac{a^{2}}{24} \partial_{i}^{2} E_{i}^{\alpha}(x)+\frac{7 a^{4}}{5760} \partial_{i}^{4} E_{i}^{\alpha}(x) .(5)$

Making use of this approximation, the classical errors arising in the kinetic Hamiltonian can be determined. To cancel these errors we take the approach of adding new terms and fixing their coefficients in order to cancel the order $a^{2}$ error. There is a great deal of freedom in choosing additional terms, which are restricted only by gauge invariance and the need for an appropriate continuum limit.

To understand the construction of gauge invariant kinetic terms, recall that the electric field and link operator transform as follows under a local gauge transformation $\Lambda(x)$ :

$E_{i}(x) \rightarrow \Lambda(x) E_{i}(x) \Lambda^{\dagger}(x)$

$U_{i}(x) \rightarrow \Lambda(x) U_{i}(x) \Lambda^{\dagger}(x+a i)$.

Consequently, the next most complicated gauge invariant term we can construct (after
$\left.\operatorname{Tr} E^{L} E^{L}\right)$ couples nearest neighbor electric fields, $\operatorname{Tr}\left[E_{i}^{L}(x) U_{i}(x) E_{i}^{L}(x+a i) U_{i}^{\dagger}(x)\right]$ and leads to the simplest improved kinetic Hamiltonian:

$$
\begin{aligned}
K_{2}= & \frac{a^{3}}{2} \sum_{x, i} \operatorname{Tr}\left[X E_{i}^{L}(x) E_{i}^{L}(x)\right. \\
& \left.+Y E_{i}^{L}(x) U_{i}(x) E_{i}^{L}(x+a i) U_{i}^{\dagger}(x)\right] .
\end{aligned}
$$

To obtain the correct continuum limit and cancel the order $a^{2}$ errors we must set $X=5 / 6$ and $Y=1 / 6$. Incorporating the interaction of arbitrarily distant links leads to an alternative improved kinetic Hamiltonian:

$$
\begin{aligned}
& K_{2}^{(n)}=\frac{a^{3}}{2} \sum_{x, i} \operatorname{Tr}\left[\left(1-\frac{1}{6 n^{2}}\right) E_{i}^{L}(x) E_{i}^{L}(x)\right. \\
& \left.+\frac{1}{6 n^{2}} E_{i}^{L}(x) U_{x \rightarrow x+n i a} E_{i}^{L}(x+n i a) U_{x+n i a \rightarrow x}\right] .
\end{aligned}
$$

An order $a^{4}$ classically improved kinetic term is given by

$$
\begin{aligned}
& K_{4}=\frac{a^{3}}{2} \sum_{x, i} \operatorname{Tr}\left[\frac{97}{120} E_{i}^{L}(x) E_{i}^{L}(x)\right. \\
& \quad+\frac{1}{5} E_{i}^{L}(x) U_{i}(x) E_{i}^{L}(x+a i) U_{i}^{\dagger}(x) \\
& \left.-\frac{1}{120} E_{i}^{L}(x) U_{x \rightarrow x+2 a i} E_{i}^{L}(x+2 a i) U_{x+2 a i \rightarrow x}(x)\right] .
\end{aligned}
$$

Further details are presented in reference [7].

\section{Variational calculations}

We are in the process of performing variational calculations using improved Hamiltonians. In such calculations we make use of the trial state:

$\left|\phi_{0}\right\rangle=\exp \left(C \operatorname{Re} S_{p}+\frac{D}{2} \operatorname{Re} S_{r}\right)|0\rangle$.

Here $S_{p}\left(S_{r}\right)$ is the sum of traced plaquettes (rectangles) on the lattice. The variational parameters $C$ and $D$ are determined, for a given coupling, by minimizing the energy density (expectation value of the improved Hamiltonian per plaquette):

$$
\begin{aligned}
\epsilon_{2} & =\langle\downarrow\rangle\left(\frac{5}{12} \frac{N^{2}-1}{\beta} C-\frac{10 \beta}{3 N}\right) \\
+\langle\bigsqcup\rangle\left(\frac{2}{3} \frac{N^{2}-1}{\beta} D+\frac{\beta}{3 N}\right)+3 \beta & (12)
\end{aligned}
$$


Here $\beta=N / g^{2}$. For the case of $\mathrm{SU}(2)$ in $2+1$ dimensions with $D=0$, it is possible to express the expectation values analytically in terms of modified Bessel functions $\left(I_{n}\right)$, leading to:

$\epsilon_{2}=\left(\frac{5 C}{2 \beta}-\frac{10 \beta}{3}\right) \frac{I_{2}(4 C)}{I_{1}(4 C)}+\frac{\beta}{3}\left(\frac{I_{2}(4 C)}{I_{1}(4 C)}\right)^{2}+3 \beta$.

This is to be compared with the standard unimproved result of Arisue, Kato and Fujiwara [8]:

$\epsilon_{0}=\left(\frac{3 C}{\beta}-2 \beta\right) \frac{I_{2}(4 C)}{I_{1}(4 C)}+2 \beta$

From the results of Eqs. (13) and (14), we calculate the respective specific heats, $C_{0}=$ $-\partial^{2} \epsilon_{0} / \partial^{2} \beta$ and $C_{2}^{(1)}$. Similar results, $C_{2}^{(n)}$ and $C_{4}$ are easily obtained from Eqs. (9) and (10) respectively. The results are shown in figure 11.

The transition region from the strong to weak coupling phase is indicated by the location of the peak [9]. As one would expect, the improved specific heats have peaks at a larger coupling $g$ than the unimproved case. It is also clear that incorporating interactions between distant links in the kinetic term does not significantly alter the degree of improvement for the case of $D=0$. For the case of $D \neq 0$, the expectation values in Eq. (12) must be calculated numerically. The same is true for $3+1$ dimensional and $\mathrm{SU}(3)$ calculations, which are currently in progress.

\section{Conclusion}

We have demonstrated the direct improvement of the Kogut-Susskind Hamiltonian by deriving a number of improved lattice Hamiltonians. Improvement has been demonstrated for the simple case of $\mathrm{SU}(2)$ in $2+1$ dimensions, by observing a transition from strong to weak coupling at a larger coupling (corresponding to a larger lattice spacing) for various improved Hamiltonians. Variational calculations of $\mathrm{SU}(2)$ and $\mathrm{SU}(3)$ massgaps in $2+1$ and $3+1$ dimensions using improved Hamiltonians are in progress.

\section{REFERENCES}

1. J. Kogut and L. Susskind, Phys. Rev. D 11, 395 (1975).

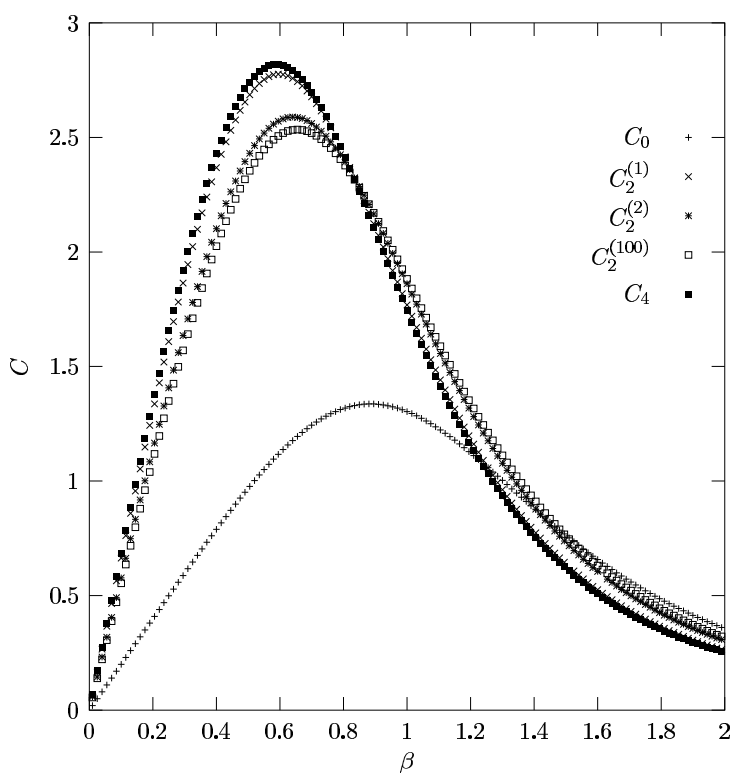

Figure 1. SU(2) specific heat in $2+1$ dimensions, calculated from various Hamiltonians. The location of the peak indicates the transition region from strong to weak coupling.

2. B. H. J. McKellar, C. R. Leonard and L. C. Hollenberg, Int. J. Mod. Phys. B 14, 2023 (2000).

3. E. B. Gregory, S. H. Guo, H. Kroger and X. Q. Luo, Phys. Rev. D 62, 054508 (2000).

4. G. P. Lepage, hep-lat/9607076.

5. X. Q. Luo, S. H. Guo, H. Kroger and D. Schutte, Phys. Rev. D 59, 034503 (1999).

6. M. Lüscher and P. Weisz, Phys. Lett. B 158, 250 (1985).

7. J. Carlsson and B. H. McKellar, Phys. Rev. D 64, 094503 (2001).

8. H. Arisue, M. Kato and T. Fujiwara, Prog. Theor. Phys. 70, 229 (1983).

9. D. Horn, M. Karliner and M. Weinstein, Phys. Rev. D 31, 2589 (1985). 